\title{
A Secure Authentication Scheme for A Public Terminal Before A Transaction
}

\author{
Chin-Ling Chen ${ }^{1}$, Yu-Yi Chen ${ }^{2}$, Jinn-Ke Jan ${ }^{3}$
}

1. Department of Computer Science and Information Engineering, Chaoyang University of Technology, Taichung, Taiwan 413, ROC. E-mail: clc@mail.cyut.edu.tw

2. Department of Management Information Systems, National Chung Hsing University, Taichung, Taiwan 402, ROC. E_mail:chenyuyi@nchu.edu.tw

3. Department of Computer Science, National Chung Hsing University, Taichung, Taiwan 402, ROC. E-mail: jkjan@cs.nchu.edu.tw

\begin{abstract}
Due to the fast progress of the Internet, and with the increasing numbers of public terminals spread everywhere, people can access personal sensitive data or perform transactions easily through these public terminals. Identifying these public terminals is therefore a most urgent topic. We propose an efficient and secure scheme that meets real environmental conditions for authenticating these public terminals before conducting a transaction.
\end{abstract}

Keywords: Cryptography, security, public terminal, kiosk

\section{Introduction}

Today, people can access personal sensitive data or perform financial transactions through public Internet kiosks that are located at malls, airports, hospitals, government agencies, etc.. Unverified kiosks are problematic for secure Internet services, as all service data is available in unencrypted form to the kiosk. Before performing personal data access or a transaction, people are required to enter their password or PINs to reliably authenticate themselves to the backend service server. However, using a public Internet access terminal creates an opportunity for persons with criminal intent to use a fake-terminal to cheat users. Currently, a counterfeit public terminal can keep users completely in the dark. The fake-terminal usually reports some plausible error messages to the users after the sensitive information has been revealed.

In accordance with these problems, in 1999, Asokan et al. [1] proposed solutions for different scenarios that correspond to different situations where the users are equipped with devices of different capacity, such as a personal trusted device with its own display, a smart card without a display, or a memory card. The Asokan et al.’s working model is shown in Fig.1. Asokan et al.'s solutions assume the use of suitable existing authentication protocols such as Secure Sockets Layer (SSL)[5], KryptoKnight [2], and Kerberos [8] Later, Cheng et al. [4] modified part of Asokan et al.'s work using the public key algorithm concept. However, in a general and comprehensive pre-view, these solutions are not perfect because many interactive steps are necessary.

In their proposals $[1,4]$, there were only four parties in their working model: the user, the personal trusted device, the public terminal and the server. Any public terminal can be activated by the user's trusted device to access various services from the server. These public terminals are susceptible to the fake-terminal attack: the attackers set up a fake terminal and steal the unsuspecting users' sensitive information, such as passwords, PINs or private e-mails, when the users attempt to use these fake terminals. Because unverified terminals are problematic for secure Internet service, all users will intend to authenticate the secure authenticated channel between the terminals and the server has already been set up before performing any transactions.

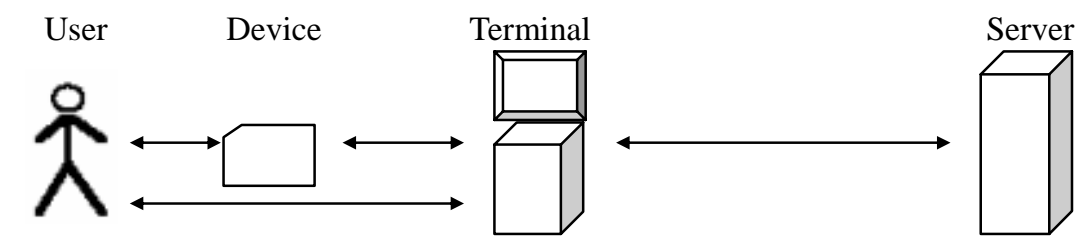

Fig. 1 Asokan et al.’s working model 
In consideration of the real environment, there should be a PoC (Point-of-Contact) server to handle all communications with kiosks [3, 6]. This concept was proposed by Laufmann [9] and the architecture was sketched as Fig. 2.

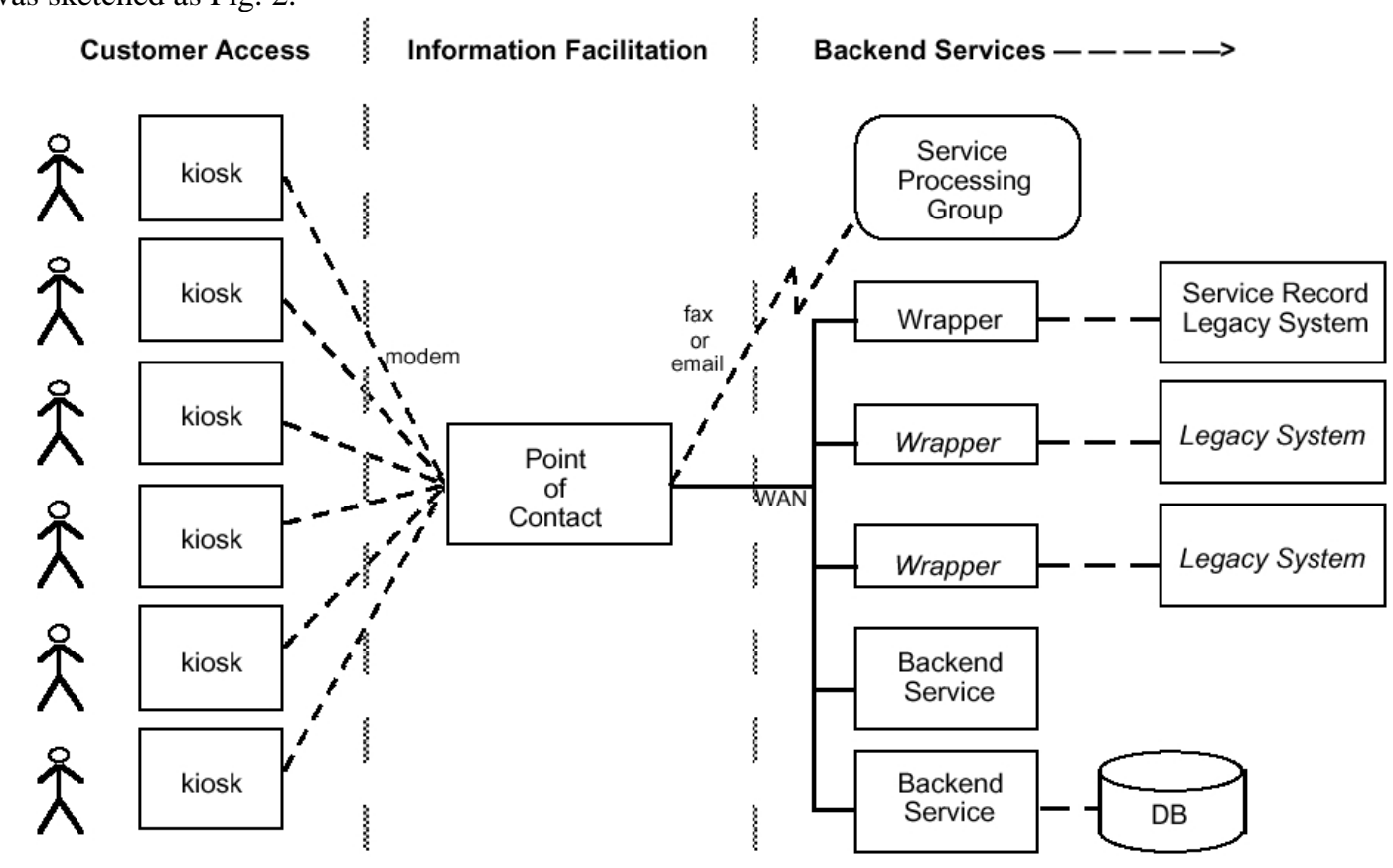

Fig. 2 Kiosk Support Service Architecture [9]

In Laufmann's proposal, the role of the PoC server is an agent to serve the deployed kiosks as a single point of contact, managing message traffic and performing service tasks between the kiosk request and the backend services. It isolates the kiosks, both physically and architecturally, from the backend service servers, and vice versa. Through the PoC server, people perform transactions to the backend service servers to access various services on the kiosks. In fact, this architecture is similar to E-voting system [7, 14]. This model is not the same as Asokan et al.'s solution. Moreover, the PoC server can only authenticate the deployed kiosk since the users' accounts are on the various kinds of backend service servers. In fact, S. Ross et al. [12] also agreed and proposed a similar architecture to bridge the gap between the terminal and the Internet services. In consideration of such an architecture, the previous works $[1,4]$ were impractical because that they assumed that only one server could directly authenticate both the users and the public terminals. We therefore propose a novel scheme base on Laufmann's model to meet the real environment.

\section{Our scheme}

In this section, we propose an efficient and more competent secure method to meet the kiosk support service architecture. There are five entities in this system, each user $\boldsymbol{U}$ is equipped with his personal trusted device $\boldsymbol{D}$ which is used to connect the public terminal $\boldsymbol{T}$ to access the Internet service from the web-site server $\boldsymbol{W}$ through the PoC server $\boldsymbol{P}$. The following Fig. 3 will briefly sketch the interaction flow of our scheme.

$\boldsymbol{U} \rightarrow \boldsymbol{D}$ : Each user $\boldsymbol{U}$ is equipped with his own personal trusted device $\boldsymbol{D}$ which could be a cell phone, PDA, smart card, or memory card. The device $\boldsymbol{D}$ is used to connect the public terminal $\boldsymbol{T}$ to access the Internet service.

$\boldsymbol{D} \rightarrow \boldsymbol{T}$ : Before processing any transaction, the device $\boldsymbol{D}$ will request the terminal $\boldsymbol{T}$ to authenticate itself to the PoC server $\boldsymbol{P}$. At the mean time, the device $\boldsymbol{D}$ also prepares some information to ask the terminal to forward and acquire the evidence to prove that this terminal is not a fake terminal. 


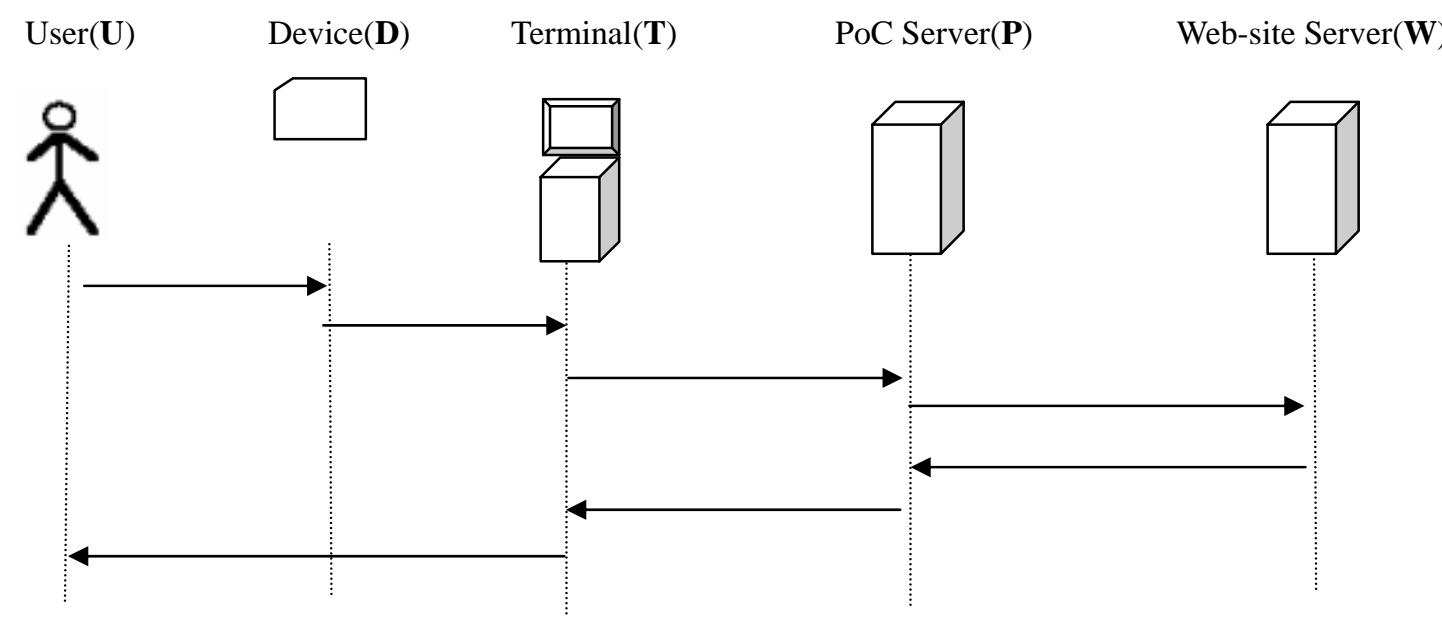

Fig. 3 The interaction flow of our scheme

$\boldsymbol{T \rightarrow \boldsymbol { P }}$ : Only a real terminal $\boldsymbol{T}$ deployed by the PoC server $\boldsymbol{P}$ can pass the authentication to forward the evidence-request information to the server $\boldsymbol{P}$.

$\boldsymbol{P} \rightarrow \boldsymbol{W}$ : Under the public key system infrastructure, the PoC server $\boldsymbol{P}$ and the web-site server $\boldsymbol{W}$ can authenticate one another. The evidence-request information can be securely forwarded to the server $\boldsymbol{W}$.

$\boldsymbol{W} \rightarrow \boldsymbol{P}$ : According to the user's account and the evidence-request information, the evidence, called authenticator, will be decrypted by the web-site server $\boldsymbol{W}$ and sent back to the PoC server $\boldsymbol{P}$.

$\boldsymbol{P} \rightarrow \boldsymbol{T}$ : The PoC server $\boldsymbol{P}$ forwards the authenticator to the terminal $\boldsymbol{T}$.

$\boldsymbol{T} \rightarrow \boldsymbol{U}$ : The terminal $\boldsymbol{T}$ shows the authenticator to the user $\boldsymbol{U}$ as evidence that it has passed the authentication series.

In addition to the above descriptions, we will use some notations to explain how to construct our scheme in the remaining part of this section.

$I D_{X} \quad$ : the identification of $\boldsymbol{X}$.

$S K_{X} \quad$ : the secret key of $\boldsymbol{X}$.

$P K_{X} \quad$ : the public key of $\boldsymbol{X}$.

$K_{U} \quad$ : the pre-defined share key between $\boldsymbol{U}$ and $\boldsymbol{W}$.

$K_{T} \quad$ : the randomly selected session key between $\boldsymbol{T}$ and $\boldsymbol{W}$.

$h($ ) : a secure and public one-way hash function. [10,11]

$E_{P K_{X}}$ () : using the public key $P K_{X}$ to encrypt some message.

$D_{S K_{X}}$ ( ) : using the secret key $S K_{X}$ to decrypt some message.

$t, t^{\prime}, t^{\prime \prime} \quad:$ the timestamps.

$\xi \quad:$ a reasonable constant time interval.

Our scheme is divided into two cases by the different types of user trusted devices: one is the device with a smart card; the other is the memory card.

\subsection{The scenario for a smart card}

\section{Step 1: $\boldsymbol{U} \rightarrow \boldsymbol{D}$}

$\boldsymbol{U}$ selects a authenticator $r_{i}$ on $\boldsymbol{D}$ and requests $\boldsymbol{D}$ to connect to $\boldsymbol{T}$. (The authenticator $r_{i}$ should be displayed if there is a built-in display on $\boldsymbol{D}$.)

Step 2: $\boldsymbol{D} \rightarrow \boldsymbol{T}$

$\boldsymbol{D}$ requests $\boldsymbol{T}$ to authenticate itself to $\boldsymbol{P}$. $\boldsymbol{D}$ then selects a nonce $N$ and sends to $\boldsymbol{T}$ the $I D_{U}, h\left(K_{U} \oplus N\right) \oplus$ authenticator ${ }_{i}, E_{P K_{W}}(N)$ data. 
Step 3: $\boldsymbol{T \rightarrow P}$

$\boldsymbol{T}$ runs a mutual authentication protocol to $\boldsymbol{P}$. If it succeeds; they set up an authenticated channel. At the same time, using $t$ as the timestamp, $\boldsymbol{T}$ also forwards the $I D_{T}, E_{P K_{W}}\left(K_{T}, t\right), I D_{U}$, $h\left(K_{U} \oplus N\right) \oplus$ authenticator $r_{i}, E_{P K_{W}}(N)$ data to $\boldsymbol{P}$ during the authentication.

Step 4: \begin{tabular}{l|}
$\boldsymbol{P} \rightarrow \boldsymbol{W}$ \\
All of the
\end{tabular}

$=E_{S K_{P}}\left(h\left(I D_{T}, E_{P K_{W}}\left(K_{T}, t\right), I D_{U}, h\left(K_{U} \oplus N\right) \oplus\right.\right.$ authenticator $\left.\left._{i}, E_{P K_{W}}(N)\right)\right)$, and $\boldsymbol{P}$ forwards all of the data with the stamp to $\boldsymbol{W}$.

Step 5. $\boldsymbol{W \rightarrow \boldsymbol { P }}$
$\boldsymbol{W}$ verifies that the stamp was really signed by $\boldsymbol{P}$ using $h\left(I D_{T}, E_{P K_{W}}\left(K_{T}, t\right), I D_{U}, h\left(K_{U} \oplus N\right) \oplus\right.$ authenticator $\left._{i}, E_{P K_{W}}(N)\right) \stackrel{?}{=} D_{P K_{P}}$ (stamp). If this is verified, $\boldsymbol{W}$ decrypts the encrypted data $E_{P K_{W}}\left(K_{T}, t\right)$ to get the session key $K_{T}$ and to check the timestamp, which should be within a reasonable range $\left(t^{\prime}-t<\xi\right) \quad W$ then decrypts the encrypted data $E_{P K_{W}}(N)$ to get the nonce $N$. Therefore, $\boldsymbol{W}$ can use the pre-defined share key $K_{U}$, the nonce $N$, the hash function $h\left(\right.$ ), and the exclusive-or function to find out the authenticator $r_{i}$ by using $h\left(K_{U} \oplus N\right) \oplus\left(h\left(K_{U} \oplus N\right) \oplus\right.$ authenticator $\left.r_{i}\right)$. Thereafter, $\boldsymbol{W}$ sends back to $\boldsymbol{P}$ the encrypted data $E_{P K_{P}}\left(I D_{T}, E_{k_{T}}\left(\right.\right.$ authenticator,$\left.\left.t^{\prime}\right)\right)$.

Step 6: $\frac{\boldsymbol{P} \rightarrow \boldsymbol{T}}{\boldsymbol{P} \text { uses }}$

uses $S K_{P}$ to decrypt the encrypted data $E_{P K_{P}}\left(I D_{T}, E_{k_{T}}\right.$ (authenticator $\left.\left.{ }_{i}, t^{\prime}\right)\right)$, and forward $E_{k_{T}}\left(\right.$ authenticator $\left.{ }_{i}, t^{\prime}\right)$ to $\boldsymbol{T}$.

Step 7: $\boldsymbol{T} \rightarrow \boldsymbol{U}$

$\boldsymbol{T}$ uses the session key $K_{T}$ to decrypt the received data $E_{k_{T}}$ (authenticator $\left.{ }_{i}, t^{\prime}\right)$ and to check the timestamp, which should be within a reasonable range $\left(t^{\prime \prime}-t^{\prime}<\xi\right)$. Consequently, $\boldsymbol{T}$ displays the authenticator $_{i}$ to $\boldsymbol{U}$. Only if the displayed authenticator $r_{i}$ is the same as the one selected by $\boldsymbol{U}$, can $\boldsymbol{U}$ trust that $\boldsymbol{T}$ has finished all of the verification procedures.

\subsection{The scenario for a memory card}

Step 1: $\boldsymbol{U} \rightarrow \boldsymbol{T}$

$\boldsymbol{U}$ requests $\boldsymbol{T}$ to authenticate itself to $\boldsymbol{P}$. $\boldsymbol{U}$ also enters the index $i$ of his random selected $E_{P K_{W}}\left(\right.$ authenticator $\left.r_{i}\right)$ which is stored in the memory card.

Step 2: $\boldsymbol{T} \rightarrow \boldsymbol{D}$

$\boldsymbol{T}$ reads the encrypted $E_{P K_{W}}$ (authenticator ${ }_{i}$ ) from $\boldsymbol{D}$.

Step 3: $\boldsymbol{T} \rightarrow \boldsymbol{P}$

$\boldsymbol{T}$ runs a mutual authentication protocol to $\boldsymbol{P}$. If it succeeds, they set up an authenticated channel. At the same time, using $t$ as the timestamp, $\boldsymbol{T}$ also forwards the $I D_{T}, E_{P K_{W}}\left(K_{T}, t\right), I D_{U}$, $E_{P K_{W}}$ (authenticator $_{i}$ ) data to $\boldsymbol{P}$ during the authentication.

Step 4: $\boldsymbol{P \rightarrow W}$

All of the above data are hashed and signed by $\boldsymbol{P}$ 's secret key $S K_{P}$ as the stamp $=E_{S K_{P}}\left(h\left(I D_{T}, E_{P K_{W}}\left(K_{T}, t\right), I D_{U}, E_{P K_{W}}\left(\right.\right.\right.$ authenticator $\left.\left.\left._{i}\right)\right)\right)$, and $\boldsymbol{P}$ forwards all of the data with the stamp to $W$.

Step 5: $\frac{\boldsymbol{W} \rightarrow \boldsymbol{P}}{\boldsymbol{W}}$ verifies if the stamp was really signed by $\boldsymbol{P}$ using $h\left(I D_{T}, E_{P K_{W}}\left(K_{T}, t\right), I D_{U}, E_{P K_{W}}\left(\right.\right.$ authentica $\left.\left.t o r_{i}\right)\right) \stackrel{?}{=} D_{P K_{P}}($ stamp $)$. If this is verified , $\boldsymbol{W}$ decrypts 
the encrypted data $E_{P K_{\mathrm{W}}}\left(K_{T}, t\right)$ to get the session key $K_{T}$ and to check the timestamp that should be within a reasonable range $\left(t^{\prime}-t<\xi\right)$. $\boldsymbol{W}$ then decrypts the encrypted data $E_{P K_{W}}\left(\right.$ authenticator $\left._{i}\right)$ to get the authentica tor $r_{i}$. Thereafter, $\boldsymbol{W}$ sends back to $\boldsymbol{P}$ the $E_{P K_{P}}\left(I D_{T}, E_{k_{T}}\right.$ (authenticator $\left.\left.{ }_{i}, t^{\prime}\right)\right)$.

Step 6: $\boldsymbol{P \rightarrow T}$

$\boldsymbol{P}$ uses $S K_{P}$ to decrypt the encrypted data $E_{P K_{P}}\left(I D_{T}, E_{k_{T}}\right.$ (authenticator,$\left.\left.t^{\prime}\right)\right)$, and forward $E_{k_{T}}$ (authenticator $\left.{ }_{i}, t^{\prime}\right)$ to $\boldsymbol{T}$.

Step 7: $\boldsymbol{T} \rightarrow \boldsymbol{U}$

$\boldsymbol{T}$ uses the session key $K_{T}$ to decrypt the received data $E_{k_{T}}$ (authenticator $\left.{ }_{i}, t^{\prime}\right)$ and to check the timestamp, which should be within a reasonable range $\left(t^{\prime \prime}-t^{\prime}<\xi\right)$. Consequently, $\boldsymbol{T}$ displays the authenticator $_{i}$ to $\boldsymbol{U}$. Only if the displayed authenticator $r_{i}$ is the same as the one selected by $\boldsymbol{U}$, can $\boldsymbol{U}$ trust that $\boldsymbol{T}$ has finished all of the verification procedures.

\section{Discussions}

In this section, we will examine our scheme against the "fake terminal attack" and the "fake-in-the middle attack". The fake terminal attack involves an attacker setting up a fake terminal to fool users into trusting it. The fake-terminal-in-the-middle attack involves a fake terminal connected to a legal terminal to fool users into trusting it.

\subsection{The smart card model}

We know that the trustworthiness of $\boldsymbol{T}$ depends on the correctness of the displayed authenticator. In our solution for the smart card, only the legal terminal $\boldsymbol{T}$ can get the correct authenticator from $\boldsymbol{W}$ through $\boldsymbol{P}$ (in step 5 and step 6) under the situation that $\boldsymbol{P}$ is authenticated by $\boldsymbol{W}$ (in step 5) and $\boldsymbol{T}$ is authenticated by $\boldsymbol{P}$ (in step 3).

To speak in detail, in step 5, $\boldsymbol{W}$ authenticates $\boldsymbol{P}$ by verifying its signature as $h\left(I D_{T}, E_{P K_{W}}\left(K_{T}, t\right), I D_{U}, h\left(K_{U} \oplus N\right) \oplus\right.$ authenticator $\left._{i}, E_{P K_{W}}(N)\right) \stackrel{?}{=} D_{P K_{P}}$ (stamp) under the public key system infrastructure. Thereafter, $\boldsymbol{P}$ will be a trusted intermediary between $\boldsymbol{W}$ and $\boldsymbol{T}$. Of course, $\boldsymbol{W}$ will also trust $\boldsymbol{T}$ since any fake terminal cannot pass the authentication to $\boldsymbol{P}$ in step 3. Using these authenticated channels between $\boldsymbol{W}$ and $\boldsymbol{T}$, the encrypted data of $I D_{U}, h\left(K_{U} \oplus N\right) \oplus$ authenticator ${ }_{i}$, and $E_{P K_{W}}(N)$ which are generated by $\boldsymbol{D}$ can be securely transferred to $\boldsymbol{W}$ in step 3 and step 4 . Only $\boldsymbol{W}$ can decrypt $h\left(K_{U} \oplus N\right) \oplus\left(h\left(K_{U} \oplus N\right) \oplus\right.$ authenticator $\left._{i}\right)=$ authenticator $_{i}$ since: (1) the nonce $N$ can be only decrypted by $\boldsymbol{W}$ using its secret key $S K_{W}$; (2) the shared key $K_{U}$ is only known to $\boldsymbol{W}$ and $\boldsymbol{U}$. In step 5, $\boldsymbol{W}$ sends back to $\boldsymbol{T}$ the encrypted data $E_{k_{T}}$ (authenticator $\left.{ }_{i}, t^{\prime}\right)$ through $\boldsymbol{P}$. Finally, in step 7, only $\boldsymbol{T}$ can decrypt it to get the authenticator $r_{i}$ using its selected session key $K_{T}$ and then show it to $\boldsymbol{U}$. Clearly, a fake terminal cannot acquire the decrypted authenticator $r_{i}$ even it gets $h\left(K_{U} \oplus N\right) \oplus$ authenticator $_{i}$ from the smart card, because it cannot pass the authentication series. In this way, any user can make insure that $\boldsymbol{T}$ is legal before any transaction. Consequently, our scheme can stand against the "fake terminal attack".

Moreover, in the "fake-terminal-in-the-middle-attack", is there any real probability for a fake terminal to be connected to a legal terminal tries to foil users in trusting it? In fact, the displayed authenticator shows only on the monitor of the legal terminal without any other output channel. Suppose the attacker cannot capture the magnetic radiation from the legal terminal or there is no place to hide a video camera in front of the legal terminal, then there is no way to clone the displayed authenticator. Clearly, the "fake-terminal-in-the-middle-attack" will not occur if the hardware and software of the legal terminal are invulnerable.

\subsection{The memory card model}

Similarly, in our solution for the memory card, only the legal terminal $\boldsymbol{T}$ can get the correct authenticator from $\boldsymbol{W}$ through $\boldsymbol{P}$ (in step 5 and step 6) under the situation when $\boldsymbol{P}$ is authenticated by $\boldsymbol{W}$ 
(in step 5) and $\boldsymbol{T}$ is authenticated by $\boldsymbol{P}$ (in step 3). In detail, $\boldsymbol{W}$ authenticates $\boldsymbol{P}$ in step 5 by verifying its signature as $h\left(I D_{T}, E_{P K_{W}}\left(K_{T}, t\right), I D_{U}, E_{P K_{W}}\left(\right.\right.$ authenticator $\left.\left._{i}\right)\right) \stackrel{?}{=} D_{P K_{P}}($ stamp $)$ under the public key system infrastructure. Thereafter, $\boldsymbol{P}$ will be a trusted intermediary between $\boldsymbol{W}$ and $\boldsymbol{T}$. Of course, $\boldsymbol{W}$ will also trust $\boldsymbol{T}$ since any fake terminal cannot pass the authentication to $\boldsymbol{P}$ in step 3. Using these authenticated channels between $\boldsymbol{W}$ and $\boldsymbol{T}$, the encrypted data of $E_{P K_{W}}$ (authenticator ${ }_{i}$ ) which is stored in the memory card can be securely transferred to $\boldsymbol{W}$ in step 3 and step 4 . Only $\boldsymbol{W}$ can decrypt it to get the authenticator $r_{i}$ using its secret key $S K_{W}$. In step $5, \boldsymbol{W}$ then sends back to $\boldsymbol{T}$ the encrypted data $E_{k_{T}}$ (authenticator ${ }_{i}, t^{\prime}$ ) through $\boldsymbol{P}$.

Finally, in step 7, only $\boldsymbol{T}$ can decrypt it to get the authenticator ${ }_{i}$ using its selected session key $K_{T}$ and then show it to $\boldsymbol{U}$. Clearly, a fake terminal cannot acquire the decrypted authenticator $r_{i}$ even it gets $E_{P K_{W}}$ (authenticator ${ }_{i}$ ) from the memory card, because it cannot pass the authentication series. In this way, our scheme against the "fake terminal attack" and the "fake-terminal-in the-middle-attack".

\section{Conclusions}

In this paper, a novel authentication model that guards against the fake terminal and fake-terminal-in-the-middle attacks for the public computer kiosk (public terminal) support service architecture. In our scheme, unverified terminals will not be problematic for secure Internet service because all users will authenticate the secure authenticated channel between the terminals and the server that has already been set up before performing any transactions. We believe that our work in this area will help bring more commerce applications to the public terminal support service architecture. For example, Smith and Pedersen [13] organized such electronic services into security taxonomies: $\mathrm{K}_{0}$ (standalone), $\mathrm{K}_{1}$ (networked), $\mathrm{K}_{2}$ (private information), $\mathrm{K}_{3}$ (transactional). According to these taxonomies, there are more security topics for further research such as:

1. How to provide private, remote information such as a user's query about current credit limits etc. via a networked kiosk system?

2. How to allow clients to change private, remote information such as electronic transaction via a networked kiosk system?

In the future, people will be accustomed to accessing various transactions through public terminals conveniently. Research on security problems should therefore receive more attention.

\section{References}

1. N. Asokan, H. Debar, M. Steiner, and M. Waidner, Authenticating public terminates, Computer Networks 31, (1999)861-970.

2. R. Bird, I. Gopal, A. Herzberg, P. Janson, S. Kutten, R. Molva, and M. Yung, Systematic design of a family of attack resistant authentications, IEEE Journal on Selected Areas in Communications 11 (5), (1993)679-693.

3. Mark W. Burris and Michael C. Pietrzyk, Interactive Transportation Information Stations, Center for Urban Transportation Research, College of Engineering, University of South Florida, http://citeseer.nj.nec.com/278953.html, (1997).

4. C. Y. Cheng, K. Seman, and J. Yunus, Authentication public terminals with smart cards, In: Proc. TENCON 2000, (2000)I-527-I-529.

5. A.O. Frier, P. Kariton, and P.C. Kocher, The SSL Protocol: Version 3.0, Technical Report, Internet Draft, (1996).

6. J. Hochnerg, S. W. Smith, M. Murphy, P. Pedersen, and B. Yantis, “Kiosk Security Handbook”, Los Alamos Unclassified Release LA-UR-95-1657, Los Alamos National Laboratory, (1995).

7. Jinn-Ke Jan, Yu-Yi Chen and Chin-Ling Chen, A Realistic Secure Anonymous E-Voting Protocol Based on the ElGamal Scheme, Proceedings of the International Conference on Communications \& Broadband Networking, Bangalore, INDIA, (2003) Sect III 1-9.

8. J. T. Kohl, and B. C. Neuman, The Kerberos Network Authentication Service (V5), Internet Request for Comment RFC 1510, (1993).

9. Steven C. Laufmann, Toward Agent-Based Software Engineering for Information-Dependent 
Enterprise Applications, In IEE Proceedings of Software Engineering, http://citeseer.ist.psu.edu/laufmann96toward.html, (1997).

10. NIST FIP PUB 180, Secure hash standard, National Institute of Standards and Technology, US department of Commerce, DRAFT, (1993).

11. R. L. Rivest, The MD5 message -digest algorithm, RFC 1231, Internet Activities Board, Internet Privacy Task Force, (1992).

12. S. Ross, J. Hill, Y.C. Mike, A.D. Joseph, D.E. Culler, and E. A. Brewer, A Security Architecture for the Post-PC World, http://citeseer.ist.psu.edu/302477.html.

13. Sean Smith, IBM Research Paul Pedersen, Los Alamos National Laborator, Organizing Electronic Services into Security Taxonomies, Proc. of the Second USENIX workshop on Electronic Commerce, http://citeseer.ist.psu.edu/338966.html, (1996).

14. Yu-Yi. Chen, Jinn-Ke Jan and Chin-Ling Chen, The Design of a Securely Anonymous Internet Voting System, Computers \& Security Vol. 23, No. 4, (2004)330-337. 